\title{
Latent class analysis of diagnostic tests for adenovirus, Bordetella pertussis and influenza virus infections in German adults with longer lasting coughs
}

\author{
C. SOBOTZKI ${ }^{1}$, M. RIFFELMANN ${ }^{2,3}$, N. KENNERKNECHT ${ }^{3}$, C. HÜLSSE $^{4}$, \\ M. LITTMANN ${ }^{4}$, A. WHITE ${ }^{5}$, R. VON KRIES ${ }^{1}$ AND C. H. WIRSING VON \\ $\mathrm{KÖNIG}^{2 *}$ \\ ${ }^{1}$ Institut für Soziale Pädiatrie und Jugendmedizin, Ludwig-Maximilians-Universität, München, Germany \\ ${ }^{2}$ Labor: Medizin Krefeld MVZ GmbH, Krefeld, Germany \\ ${ }^{3}$ Institut für Hygiene und Labormedizin, HELIOS Klinikum Krefeld, Germany \\ ${ }_{5}^{4}$ Landesamt für Gesundheit und Soziales des Landes Mecklenburg Vorpommern, Rostock, Germany \\ ${ }^{5}$ Trinity College Dublin, School of Computer Science and Statistics, Dublin, Ireland
}

Received 18 June 2015; Final revision 18 August 2015; Accepted 26 August 2015;

first published online 18 September 2015

\section{SUMMARY}

Laboratory tests in adult outpatients with longer lasting coughs to identify a potential causal pathogen are rarely performed, and there is no gold standard for these diagnostic tests. While the diagnostic validity of serological tests for pertussis is well established their potential contribution for diagnosing adenovirus and influenza virus A and B infections is unclear. A sentinel study into the population-based incidence of longer lasting coughs in adults was done in Rostock (former East Germany) and Krefeld (former West Germany). A total of 971 outpatients who consulted general practitioners or internists were included. Inclusion criteria were coughing for $\geqslant 1$ week and no chronic respiratory diseases. We evaluated the performance of polymerase chain reaction (PCR) as well as IgG and IgA serology, applying a latent class model for diagnosing infections with adenovirus, $B$. pertussis, and influenza virus A and B. The adult outpatients first sought medical attention when they had been coughing for a median of 3 weeks. In this situation, direct detection of infectious agents by PCR had a low sensitivity. Modelling showed that additional serological tests equally improved sensitivity and specificity for diagnosis for adenovirus, B. pertussis and influenza virus A and B infections. The combination of serology and PCR may improve the overall performance of diagnostic tests for B. pertussis and also for adenovirus, and influenza virus A and B infections.

Key words: Adenovirus, adults, cough, influenza virus, latent class analysis, pertussis.

\section{INTRODUCTION}

With the advent of molecular methods in diagnostic virology, real-time polymerase chain reaction (PCR) is regarded as the method of choice for diagnosing

\footnotetext{
* Author for correspondence: Dr C. H. Wirsing von Koenig, Labor: Medizin Krefeld MVZ GmbH, Krefeld, Germany.

(Email: carlheinz.wirsingvonkoenig@helios-kliniken.de)
}

viral respiratory diseases such as influenza $\mathrm{A}$ and $\mathrm{B}$ and adenovirus infections [1]. In adult outpatients, however, symptoms may persist for a lengthy period before medical attention is sought, and consequently, even if attempted, the sensitivity of direct detection of viruses may be not sufficiently high. For infections with Bordetella pertussis in adults, various studies have demonstrated that the sensitivity of Bordetella PCR was well below $10 \%$ and thus, serology has 
been the mainstay for diagnosing these infections [2]. As no accepted 'gold standard' exists for these infections, latent class analysis (LCA) has been successfully applied to estimate the sensitivities and specificities of PCR and serology for pertussis diagnosis [3]. LCA is a well-documented and statistically valid tool in absence of a gold standard [3, 4]. Moreover, the characteristics of this method have been extensively studied in comparison with estimates where a gold standard was available [4].

We used a sentinel study into longer lasting cough in adults with almost 1000 patients in two German cities to study the application of the latent class model for the diagnosis of pertussis and to extend it to the diagnosis of adenovirus as well as influenza virus A and $\mathrm{B}$ infections in order to evaluate the relative role of viral serology in this outpatient population.

\section{PATIENTS AND METHODS}

Patients were recruited from general practitioners (GPs) and internists, who were active in general internal medicine (Allgemeinärztlich tätige Internisten) (summarized as GPs) in Krefeld (former West Germany) and Rostock (former East Germany). Between 2001 and 2004 a total of 971 patients were included in the study. The study had 21 sentinels in Rostock and 14 in Krefeld. The 21 sentinels in Rostock treated $34 \cdot 2 \%$ of all patients in Rostock and the 14 sentinels in Krefeld treated $19 \cdot 2 \%$ of patients there. This study was approved by the ethical committees of the medical boards in Krefeld (Ärztekammer Nordrhein) and Rostock (Ärztekammer MecklenburgVorpommern).

Inclusion criteria. Adult patients with a cough of $\geqslant 7$ days' duration were eligible for inclusion. Patients treated for chronic respiratory diseases such as chronic obstructive pulmonary disease, and patients participating in other studies were excluded. Clinical data about symptomatology, other diseases, contacts, professional background, duration of coughing and days off work were collected by two questionnaires. The first questionnaire asked about the duration of coughing before the first visit, for various other symptoms, for a history of similar episodes and for a contact anamnesis.

A total of 971 patients were included (455 from Rostock, 516 from Krefeld). Sixty-seven per cent were female $(n=650)$ and $33 \%$ were male $(n=221)$. The female:male ratio in Rostock was 71:29, and in
Krefeld 65:35 $(P>0 \cdot 2)$. The age distribution of all patients is given in Figure 1.

A total of 934 patients reported the duration of coughing symptoms before the first visit. For estimation of the mean time of symptoms before seeking medical attention we assumed that a total of 80 patients $(8 \cdot 5 \%)$ reporting symptoms for $>3$ months (12 weeks) would suffer from chronic coughing, and these were excluded from estimating the mean time before the first visit. Another 14 patients (1.5\%) reported symptoms for $<7$ days (inclusion criterion) and these were also excluded from analysis.

Diagnostic procedures. A nasopharyngeal swab, pharyngeal washing and a peripheral blood sample was taken from all patients at presentation at the sentinels. Nasopharyngeal swabs were immersed in modified Amies medium with charcoal. Pharyngeal washings were sampled into a sterile tube. All samples were transported at ambient temperature to the central laboratory (Krefeld).

Laboratory tests. Nasopharyngeal swabs were tested for Bordetella DNA by real-time PCR targeting IS481 (B. pertussis), and IS1001 (B. parapertussis) [5]. Pharyngeal washings were tested for adenovirus DNA, and influenza viruses A and B RNA by an in-house real-time PCR. For adenovirus PCR, a 131 nt fragment from the hexon gene of human adenovirus type 2 , belonging to adenovirus species $C$, was amplified using TaqMan primers and probes and the amplification was performed on a 7500 RT-PCR system (ABI Biosystems, USA) [6]. For influenza A virus a $101 \mathrm{nt}$ fragment from the $\mathrm{M}$ gene was amplified. The reverse primer was degenerated and a specific primer for the haemagglutinin variant $\mathrm{H} 1 \mathrm{sw}$ was added. The probe was tagged with an amplifier molecule (ZNA; zipped nucleic acid). For influenza B virus a $89 \mathrm{nt}$ fragment from the $\mathrm{M}$ gene was amplified. Amplification was done either on a 7500 RT-PCR or StepOne system (ABI Biosystems) [7].

Serum samples were tested for $\operatorname{IgG}$ and $\mathrm{IgA}$ antibodies to adenovirus, for IgG and IgA antibodies to influenza virus $A$ and $B$ with a nucleoprotein as the respective antigen by micro-well ELISAs (all Virion Serion GmbH, Würzburg, Germany). IgG and IgA antibodies to pertussis toxin (PT) were measured by an in-house ELISA [8].

Interpretation of serology results. The commercial ELISAs measured antibodies quantitatively in arbitrary unit (AU/ml). The sensitivity of the commercial 


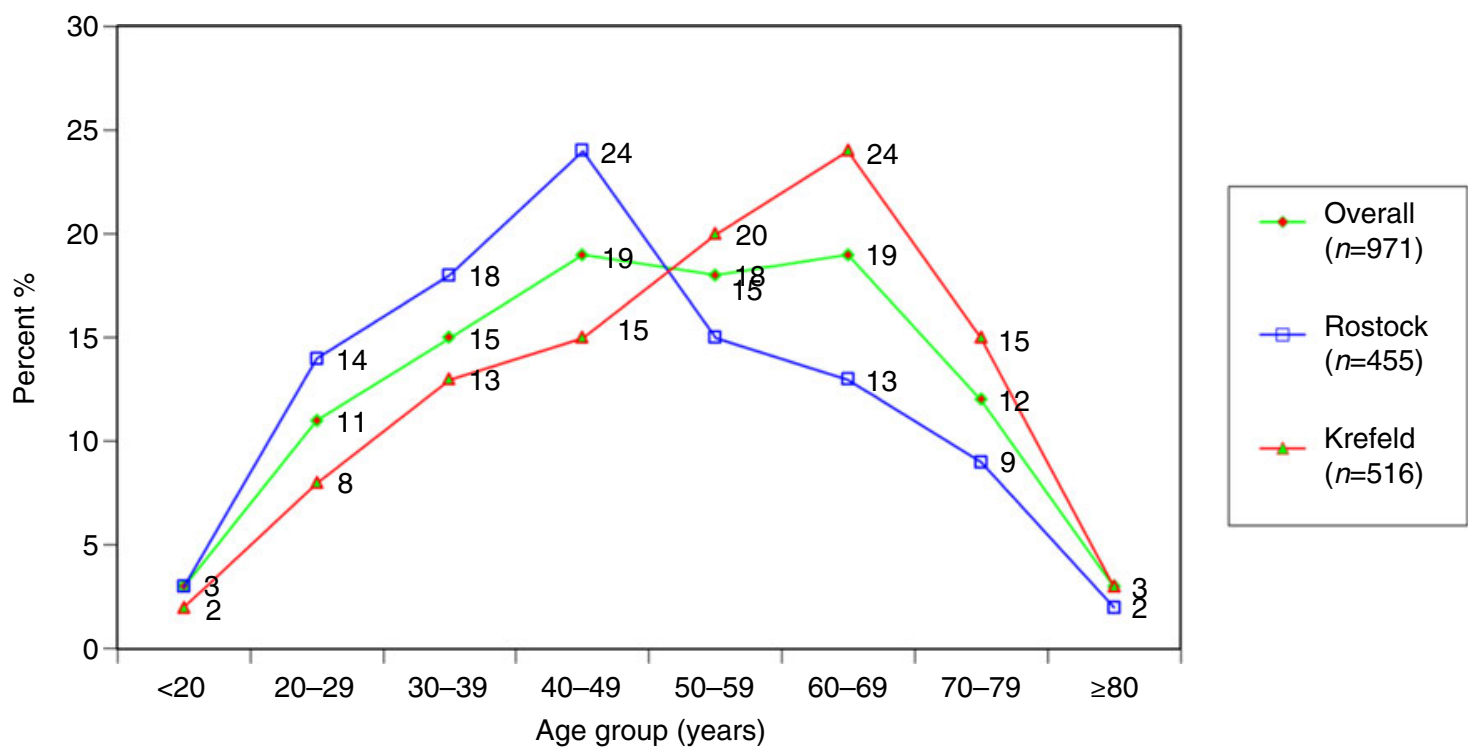

Fig. 1. Age distribution of study patients.

test systems was set to detect no antibodies to influenza virus $\mathrm{A}$ and $\mathrm{B}$ in healthy blood donors, irrespective of their immunization status to influenza virus. For antibodies to adenovirus, $95 \%$ of obviously healthy blood donors had no antibodies detected. Thus, we defined all detectable antibody levels as possibly indicative of a recent contact. For IgG anti-PT we followed the recommendations of the European reference laboratories [9] and $\geqslant 100 \mathrm{IU} / \mathrm{ml}$ was regarded as indicative of recent contact. For IgA anti-PT $\geqslant 12 \mathrm{IU} / \mathrm{ml}$ was regarded as indicative of recent contact.

\section{Statistical evaluation}

We used LCA to assess diagnostic test accuracy [10]. LCA hypothesizes the existence of one or more unobserved categorical variables to explain the relationships between a set of observed categorical variables. We treat the latent variable which corresponds to multiple positive diagnoses as the unobserved true disease status of the patients [11]. In the medical diagnosis context, the observed variables are, e.g. test results, usually dichotomized into a binary classification (positive and negative).

In the LCA model a posterior probability of the infections of interest is estimated with a frequentist expectation-maximization (EM) algorithm proposed by $\mathrm{Qu}$ et al. [12]. For each possible test combination a response pattern depicting the posterior probability of being infected was calculated. If the posterior probability was $>0 \cdot 50$, subjects were classified as infected [3]. We repeated each LCA several times with different starting values for the EM algorithm in order to identify a stable pattern as proposed by Beath [13]. Sensitivity and specificity of the individual tests were estimated from the LCA. The sensitivity is the probability of the respective test to identify the subject correctly as infected under the condition that the subject is infected. Specificity on the other hand is the probability of correctly identifying a subject as not infected. Since the true infection status is unknown the latent class method constructs a hypothetical standard, and assesses the individual test's performance with respect to this hypothetical standard [13]. The $95 \%$ confidence limits were calculated using bootstrap methods [14].

As a sensitivity analysis we applied Bayesian LCA with non-informative priors via EM algorithms and Gibbs sampler [15], a Markov chain Monte Carlo (MCMC) method. For the Gibbs sampler a sufficient burn-in was chosen and thinning was used to reduce autocorrelation between the observed output terms $[16,17]$. An overview of the applied methods is described in Gentle et al. [18].

All analyses were performed with statistical software $\mathrm{R}$ 3.1.2 [19] and the add-on packages randomLCA [20] and BayesLCA [15].

\section{RESULTS}

From the 934 patients, who reported the duration of coughing symptoms, we assumed that 80 had chronic cough (duration $>12$ weeks), and 14 reported coughing 
Table 1. Overall test results for polymerase chain reaction (PCR) and serology

\begin{tabular}{llc}
\hline \hline & $\begin{array}{l}\text { Negative } \\
\text { test result } \\
n(\%)\end{array}$ & $\begin{array}{l}\text { Positive } \\
\text { test result } \\
n(\%)\end{array}$ \\
Test & & \\
\hline B. pertussis & $909(95 \cdot 89)$ & $39(4 \cdot 11)$ \\
PT IgG & $884(93 \cdot 25)$ & $64(6 \cdot 75)$ \\
PT IgA & $937(98 \cdot 84)$ & $11(1 \cdot 16)$ \\
Bp PCR & & $123(12 \cdot 96)$ \\
Adenovirus & $826(87 \cdot 04)$ & $176(18 \cdot 55)$ \\
Adeno IgG & $773(81 \cdot 45)$ & $23(2 \cdot 42)$ \\
Adeno IgA & $926(97 \cdot 58)$ & $47(4 \cdot 95)$ \\
Adeno PCR & $902(95 \cdot 05)$ & $132(13 \cdot 91)$ \\
Influenza A & $817(86 \cdot 09)$ & $11(1 \cdot 16)$ \\
InfA IgG & $938(98 \cdot 84)$ & $244(25 \cdot 71)$ \\
InfA IgA & & $57(6 \cdot 01)$ \\
InfA PCR & $705(74 \cdot 29)$ & $8(0 \cdot 84)$ \\
Influenza B & $892(93 \cdot 99)$ & \\
InfB IgG & $941(99 \cdot 16)$ & \\
InfB IgA & & \\
InfB PCR &
\end{tabular}

PT IgG, IgG antibodies to pertussis toxin (PT); PT IgA, IgA antibodies to PT; Bp PCR, PCR for detecting Bordetella DNA; Adeno IgG, IgG antibodies to adenovirus; Adeno IgA, IgA antibodies to adenovirus; Adeno PCR, PCR for detecting adenovirus DNA; InfA IgG, IgG antibodies to influenza virus $\mathrm{A}$; $\operatorname{InfA} \operatorname{IgA}, \operatorname{IgA}$ antibodies to influenza virus A; InfA PCR, PCR for detecting influenza virus A RNA; InfB IgG, IgG antibodies to influenza virus B; InfB IgA, IgA antibodies to influenza virus B; InfB PCR, PCR for detecting influenza virus B RNA.

for $<1$ week. The remaining 840 patients reported coughing for a mean of $25 \cdot 8 \pm 17 \cdot 6$ days ( 3.7 weeks) and for a median of 21 days [ $95 \%$ confidence interval (CI) 7-63 days] before first seeking medical attention. No statistically significant difference was observed in the duration of symptoms between Krefeld and Rostock $(P>0 \cdot 1)$.

Of the 971 study subjects, 948 patients had three tests for pertussis and 949 had three tests for adenovirus and influenza $\mathrm{A}$ and $\mathrm{B}$, respectively.

\section{Pertussis}

A positive test result for pertussis was observed in 39 subjects $(4 \cdot 1 \%)$ by PT IgG, in 64 subjects $(6 \cdot 8 \%)$ by PT IgA and in 11 subjects $(1.2 \%)$ by B. pertussis PCR (Table 1). Based on the LCA model a total of 23 subjects were classified as infected (Supplementary Table S1). According to this model, $22(18+4)$ out of $23(96 \%)$ could be detected with the test combination of PT IgG and PT IgA. Based on the LCA model the sensitivity for PT IgG was estimated as 1.00 (95\% CI
Table 2. Estimated sensitivity and specificity of indicators of pertussis, adenovirus and influenza according to latent class analysis

\begin{tabular}{|c|c|c|}
\hline Test & Sensitivity & Specificity \\
\hline \multicolumn{3}{|l|}{ B. pertussis } \\
\hline PT IgG & $1.00(0 \cdot 99-1 \cdot 00)$ & $0.99(0.67-1.00)$ \\
\hline PT IgA & $0 \cdot 81(0 \cdot 00-1 \cdot 00)$ & $0.95(0.89-0.98)$ \\
\hline Bp PCR & $0.19(0.04-0.53)$ & $0.99(0.93-1.00)$ \\
\hline \multicolumn{3}{|l|}{ Adenovirus } \\
\hline Adeno IgG & $0.39(0.01-0.93)$ & $0.93(0.13-1 \cdot 00)$ \\
\hline Adeno IgA & $1 \cdot 00(0 \cdot 41-1 \cdot 00)$ & $1.00(0.99-1.00)$ \\
\hline Adeno PCR & $0.05(0 \cdot 00-0.43)$ & $0.98(0.96-0.99)$ \\
\hline \multicolumn{3}{|l|}{ Influenza A } \\
\hline $\operatorname{InfA} \operatorname{IgG}$ & $0 \cdot 99(0 \cdot 21-1 \cdot 00)$ & $1 \cdot 00(0 \cdot 99-1 \cdot 00)$ \\
\hline $\operatorname{InfA} \operatorname{IgA}$ & $0 \cdot 21(0 \cdot 00-0 \cdot 99)$ & $0 \cdot 86(0 \cdot 00-1 \cdot 00)$ \\
\hline InfA PCR & $0 \cdot 02(0 \cdot 00-1 \cdot 00)$ & $0.99(0.44-1.00)$ \\
\hline \multicolumn{3}{|l|}{ Influenza B } \\
\hline InfB IgG & $1 \cdot 00(0 \cdot 99-1 \cdot 00)$ & $0.76(0.04-0.99)$ \\
\hline $\operatorname{InfB} \operatorname{Ig} A$ & $1.00(0.98-1.00)$ & $0.96(0.06-1.00)$ \\
\hline InfB PCR & $0 \cdot 10(0 \cdot 00-1 \cdot 00)$ & $0.99(0.96-1.00)$ \\
\hline
\end{tabular}

Values in parentheses are $95 \%$ confidence intervals.

For abbreviations see Table 1.

0.99-1.00) followed by PT IgA $0.81(95 \%$ CI 0.00 $1 \cdot 00)$ and B. pertussis PCR 0.19 (95\% CI 0.04-0.53) (Table 2). The specificity was high in all tests. The data presented in Table 2 are based on the frequentist EM algorithm. A Bayesian EM algorithm and the Gibbs sampler yielded identical classification of pertussis infection (data not shown).

\section{Adenovirus}

A positive test result for adenovirus was observed in 123 subjects $(13.0 \%)$ by adenovirus $\mathrm{IgG}$, in $176 \mathrm{sub}-$ jects $(18.5 \%)$ by adenovirus IgA and in 23 subjects $(2 \cdot 4 \%)$ by adenovirus PCR (Table 1). Based on the LCA model a total of 176 subjects were classified as infected (Supplementary Table S2). According to this model, all infected subjects could be detected by adenovirus IgA. The highest sensitivity was estimated as $1.00(95 \%$ CI $0 \cdot 99-1 \cdot 00)$ for adenovirus IgA, $0 \cdot 81$ (95\% CI $0.00-1 \cdot 00$ ) for adenovirus IgG and $0 \cdot 19$ (95\% CI 0.04-0.53) for adenovirus PCR (Table 2). The specificity was high in all tests. The Bayesian EM algorithm and the Gibbs sampler yielded identical classification of adenovirus infection as the frequentist EM algorithm (data not shown).

\section{Influenza A}

A positive test result for influenza A was observed in 47 subjects $(5 \cdot 0 \%)$ by influenza $\mathrm{A} \operatorname{IgG}$, in 132 subjects 
(13.9\%) by influenza A IgA and in 11 subjects $(1 \cdot 2 \%)$ by influenza A PCR (Table 1). Based on the LCA model a total of 47 subjects were classified as infected, all detected by influenza A IgG (Supplementary Table S3). Low sensitivity was estimated with $0 \cdot 21$ (95\% CI 0.00-0.99) for influenza A IgA and 0.02 (95\% CI 0.00-1.00) for influenza A PCR (Table 2). Both Bayesian LCA models yielded the same predicted group membership for influenza A (data not shown).

\section{Influenza B}

For influenza B a positive test result was observed in 244 subjects $(25.7 \%)$ by influenza $\mathrm{B}$ IgG, in 57 subjects $(6 \cdot 0 \%)$ by influenza B IgA and in eight subjects $(0 \cdot 8 \%)$ by influenza B PCR (Table 1). In the estimated LCA model for influenza B a total of 29 subjects were classified as infected by both influenza B IgG and influenza B IgA (Supplementary Table S4). A very low sensitivity with $0 \cdot 10(95 \%$ CI $0 \cdot 00-1 \cdot 00)$ was estimated for influenza B PCR (Table 2). Influenza B IgG had a high false-positive rate resulting in a moderate specificity. Both Bayesian LCA models yielded the same predicted group membership for influenza B (data not shown).

\section{DISCUSSION}

In this cohort of adult patients in Germany who were coughing for $\geqslant 7$ days before seeking medical attention, the combination of serology and PCR seemed not only to improve the overall performance of diagnostic tests for $B$. pertussis but also for adenovirus, and influenza virus A and B infections.

For interpretation of our data some points of our study design are worth discussing: first, our inclusion criteria selected against detecting influenza-like illnesses, as the clinical definition of influenza would normally require an acute onset with high fever and pronounced malaise. In our study setting we found that patients were coughing for a median of 3 weeks before visiting a physician. Consequently, we assume that the detection of viral RNA for influenza virus $\mathrm{A}$ and $\mathrm{B}$, as well as viral DNA for adenovirus may have a similarly low sensitivity as has been documented in adult pertussis cases for detection of Bordetella DNA [2, 5]. In this respect, our study reproduced the assumption made in an earlier study using LCA [3]. Our pre-analytics, however, may have additionally disadvantaged the detection of viral nucleic acids, as we used oropharyngeal washings that were found to be less sensitive than nasopharyngeal samples [21]. In that study, it was observed that in 550 hospitalized patients, viral RNA/DNA could be detected in 136 oropharyngeal swabs, and also in 184 nasopharyngeal swabs. However, at the time of our study, our protocol depended on oropharyngeal washings. Furthermore, the diagnostic gold standard is also a function of age. Whereas in adults, sensitivity of PCR is rather low, it can be around 70\% in infants or young children who visit their physician/paediatrician early after the onset of cough [5].

The specificity of the antigens used in diagnostic serology may be another reason for concern. In pertussis serology, recommendations about the use of specific antigens have been published [9], but many serological kits still need to evolve and follow the consensus recommendations, which is not always the case. This is a major problem not only for routine biological diagnosis but also epidemiological studies. In influenza serology, and even less so in adenoviral serology, no consensus about antigens useful in the diagnosis of respiratory disease has been achieved. The commercial ELISA systems employed in this study used nucleoproteins for influenza A and B serology and hexon protein for adenovirus serology. For influenza serology these tests have been successfully used in diagnostic serology [22] with a good sensitivity and specificity. For adenovirus respiratory infections in non-immunocompromised adults, isotype-specific ELISA systems were only rarely used [23], but displayed sufficient sensitivity and specificity.

We deliberately did not confine the analyses to cases with confirmed coughing between 7 days and 3 months. Instead we chose an 'intention to diagnose' approach to be as close as possible to the physicians' diagnostic perspective. According to the physician, all patients had met the predefined case definition and for these patients the potential diagnostic properties of our test combination needed to be established.

The LCA model used here is a well-documented and statistically valid tool in the absence of a gold standard [3, 4] This approach has been used in another cohort with suspected pertussis [3], but has not previously been applied to a combined set of tests for pertussis, adenovirus, and influenza $\mathrm{A}$ and $\mathrm{B}$ in a large population of adult patients with respiratory disease and cough. A methodological strength of our analysis is the validation of the frequentist approach by Bayesian approaches. A methodological limitation of this approach is that a combination of only three 
tests was applied. The total number of 'true' cases identified might be greater if a further set of diagnostic tests had been be available for the LCA. Due to the limited number of tests underlying the analysis, we could not use the random-effects model proposed by $\mathrm{Qu}$ et al. [12] that integrate local dependence between tests through a single normally distributed random effect [24]. This may be a critical issue since the assumption of conditional independence is often violated in practice $[4,12,24,25]$. It has been shown that ignoring conditional independence biases the estimates of diagnostic accuracy. Even under study conditions, however, a combination of more than three tests is very unusual, and in clinical diagnostic practice diagnostic tests for outpatients with chronic coughs are rarely if ever applied. Thus, a recently published clinical guideline on acute coughs in adults [26] only mentions pertussis when a laboratory diagnosis may be attempted.

We partly estimated wide confidence limits for the sensitivity or specificity. This could be caused by no or only a few subjects in some response patterns, leading to a very flat likelihood. In this case, the estimates will have a high variance even if the likelihood is identifiable [27].

The role of acute infections in chronic cough in adults without underlying lung disease was unknown. In a population of $>900$ individuals with clinical signs of respiratory disease and cough and without positive PCR, additional cases of $B$. pertussis, adenovirus, and influenza virus $A$ and $B$ infections were identified by serology (Table 1). For all conditions a combination of IgG and IgA serological testing had the best test performance while PCR had little overall value in this setting. Although PCR is generally regarded as the diagnostic 'gold standard', our study shows that for this patient group the addition of diagnostic viral serology may be a valuable adjunct for laboratory diagnosis. Given the constraints of the study, however, additional work is needed to prospectively define the optimal use of PCR and diagnostic serology in longer lasting coughing disease in adults.

\section{APPENDIX. KRESH Sentinel Physicians}

Krefeld: Frau und Herr Dr. Rieger, Praxis Dr. Krausbauer und Frau Dr. Möhrke, Herr Dr. Dotzel, Herr Dr. Frackenpohl, Praxis Dr. Lüdemann und Herr Dr. Schultz, Herr Dr. Urban, Herr Dr. Urlbauer, Herr Dr. Baselt, Herr Dr. Ridderskamp, Praxis Dr. Hochbruck und Dr. Hermann, Praxis Dr.
Kemmerich und Dr. Thomaßen, Herr Dr. Meißner, Herr Dr. Seelhoff.

Rostock: Frau Dr. Lusch, Herr Dr. Rothe, Frau Dr. Krüger, Herr Dr. Th. Hohlbein, Frau Dr. Borchmann, Frau Dr. Kähler, Frau Dr. Bichowski, Frau Dr. Lüder, Frau Dr. Glaser, Frau Dr. B. Hohlbein, Frau Dr. Vilbrandt, Herr Dr. Anft, Frau Dr. Burow, Frau Dr. Olwig, Frau Dr. Engelhardt, Frau Dr. Lange, Herr Dr. Langer, Frau Dr. Evert, Frau Dr. Michelsen, Frau Dr. Frank, Frau Dr. Morcan

\section{SUPPLEMENTARY MATERIAL}

For supplementary material accompanying this paper visit http://dx.doi.org/10.1017/S0950268815002149.

\section{ACKNOWLEDGEMENTS}

The recruiting of patients and the logistics in this study was funded jointly by GlaxoSmithKline Pharma GmbH \& Co. KG, Munich, Germany and Sanofi Pasteur MSD GmbH, Leimen, Germany. No funding was received for the statistical evaluation of for performing various diagnostic tests.

\section{DECLARATION OF INTEREST}

None.

\section{REFERENCES}

1. Qu JX, et al. Viral etiology of community-acquired pneumonia among adolescents and adults with mild or moderate severity and its relation to age and severity. BMC Infectious Diseases 2015; 15: 89.

2. von Konig $\mathbf{C H}$, et al. Pertussis of adults and infants. Lancet Infectious Diseases 2002; 2: 744-750.

3. Baughman AL, et al. Utility of composite reference standards and latent class analysis in evaluating the clinical accuracy of diagnostic tests for pertussis. Clinical and Vaccine Immunology 2008; 15: 106-114.

4. Rutjes AW, et al. Evaluation of diagnostic tests when there is no gold standard. A review of methods. Health Technology Assessment 2007; 11: iii, ix-51.

5. Riffelmann M, et al. Nucleic Acid amplification tests for diagnosis of Bordetella infections. Journal of Clinical Microbiology 2005; 43: 4925-4929.

6. Heim A, et al. Rapid and quantitative detection of human adenovirus DNA by real-time PCR. Journal of Medical Virology 2003; 70: 228-239.

7. Schulze M, et al. Diagnostic approach for the differentiation of the pandemic influenza $\mathrm{A}(\mathrm{H} 1 \mathrm{~N} 1) \mathrm{v}$ virus from recent human influenza viruses by real-time PCR. PLoS ONE 2010; 5: e9966. 
8. Wirsing von Konig CH, et al. Evaluation of a singlesample serological technique for diagnosing pertussis in unvaccinated children. European Journal of Clinical Microbiology \& Infectious Diseases 1999; 18: 341-345.

9. Guiso N, et al. What to do and what not to do in pertussis serology: Recommendations from the European reference laboratories. European Journal of Clinical Microbiology \& Infectious Diseases 2011; 30: 307-312.

10. Goodman LA. Exploratory latent structure analysis using both identifiable and unidentifiable models. Biometrika 1974; 61: 215-231.

11. Rindskopf D, Rindskopf $\mathbf{W}$. The value of latent class analysis in medical diagnosis. Statistics in Medicine 1986; 5: 21-27.

12. Qu Y, Tan M, Kutner MH. Random effects models in latent class analysis for evaluating accuracy of diagnostic tests. Biometrics 1996; 52: 797-810.

13. Beath K. Random LCA examples (https://cran.r-project. org/web/packages/randomLCA/vignettes/randomLCAexample.pdf). 2014.

14. Efron B. Bootstrap methods: another look at the jackknife. Annals of Statistics 1979; 7: 1-26.

15. White A, Murphy T. Bayes LCA: an R package for Bayesian latent class analysis. Journal of Statistical Software 2014; 61(13).

16. Kleinman K, Horton N. SAS and R: Data Management, Statistical Analysis, and Graphics, 2nd edn. Boca Raton, FL: CRC Press, 2014.

17. Plummer M, et al. CODA: convergence diagnosis and output analysis for MCMC. R News 2006; 6: 7-11.

18. Gentle J, Härdle W, Mori Y. Handbook of Computational Statistics: Concepts and Methods. Berlin: Springer Science \& Business Media, 2012.
19. R Development Core Team. $R: A$ Language and Environment for Statistical Computing. Vienna, Austria: R Foundation for Statistical Computing, 2011.

20. Beath K (ed.). randomLCA: Random Effects Latent Class Analysis (https://cran.r-project.org/package= randomLCA), 2015.

21. Lieberman D, et al. Identification of respiratory viruses in adults: nasopharyngeal versus oropharyngeal sampling. Journal of Clinical Microbiology 2009; 47: 3439-3443.

22. Voeten JT, et al. Use of recombinant nucleoproteins in enzyme-linked immunosorbent assays for detection of virus-specific immunoglobulin $\mathrm{A}$ (IgA) and $\operatorname{IgG}$ antibodies in influenza virus A- or B-infected patients. Journal of Clinical Microbiology 1998; 36: 3527-3531.

23. Julkunen I, Lehtomaki K, Hovi T. Immunoglobulin class-specific serological responses to adenovirus in respiratory infections of young adult men. Journal of Clinical Microbiology 1986; 24: 112-115.

24. Reboussin BA, Ip EH, Wolfson M. Locally dependent latent class models with covariates: an application to under-age drinking in the USA. Journal of the Royal Statistical Society Series A 2008; 171: 877-897.

25. Pepe MS, Janes H. Insights into latent class analysis of diagnostic test performance. Biostatistics 2007; 8: 474-484.

26. Holzinger F, et al. The diagnosis and treatment of acute cough in adults. Deutsches Arzteblatt International 2014; 111: 356-363.

27. Madans J, et al. Question Evaluation Methods: Contributing to the Science of Data Quality. Hoboken, NJ: John Wiley \& Sons, 2011. 